\title{
Research on Information Resource Sharing and Big Data of Sports Industry in the Background of OpenStack Cloud Platform
}

\author{
Chuan Mou ${ }^{1}$ and Ye Cheng $\mathbb{D}^{2}$ \\ ${ }^{1}$ College of Physical Education, Kunsan National University, Kunsan 54150, Jeonro-do, Republic of Korea \\ ${ }^{2}$ Department of Physical Education, Hebei University of Economics and Business, Shijiazhuang 050061, Hebei, China \\ Correspondence should be addressed to Ye Cheng; chengye198926@163.com
}

Received 10 May 2021; Revised 1 July 2021; Accepted 7 August 2021; Published 15 August 2021

Academic Editor: Yuan Tian

Copyright (C) 2021 Chuan Mou and Ye Cheng. This is an open access article distributed under the Creative Commons Attribution License, which permits unrestricted use, distribution, and reproduction in any medium, provided the original work is properly cited.

\begin{abstract}
The rapid development of information technology and Internet makes the sports information resources retrieval service more convenient and quick; sports policy in recent years lays a foundation for the development of the Internet + sports, the development of sports industry in the process of our country economy level of development status, and the development of sports industry into the era of information and big data. This paper takes OpenStack cloud platform as the research basis (1) to realize the sharing of sports industry information resources in OpenStack cloud technology and (2) to realize big data analysis of sports industry and (3) empirical research on big data of sports industry. The main content is to realize the construction of sports resources informatization based on the OpenStack cloud platform. Through the analysis and empirical study of the big data of the sports industry, the influence of the development of the sports industry in the process of China's economic development is discussed. In this paper, the experimental results show that the sports industry showed a positive impact in the process of economic development, the sports economy for the development of the economy, the contribution rate reached $11.77 \%$, the sports industry for the development of the economy, the pull rate of $1.056 \%$, based on the cloud platform of information resources sharing of data analysis, sports industry for the development of the economy has a positive role in promoting.
\end{abstract}

\section{Introduction}

The rapid development of economy leads to the rapid growth of data. The public resource information sharing and the healthy development of big data industry have attracted great attention of the country. At present, the total amount of data appearing and stored in China exceeds 800 exabytes, and China's e-government development index is 0.6071, ranking 63rd [1]. The development and utilization of big data resources have risen to the national level, and big data technology has been applied to various fields. Government departments put forward open public data resources sharing, improve big data infrastructure construction and development of big data in various fields of application, the formation of large data product system, perfect the big data industry chain, and improve the big data security system, to ensure that big data [2].
In the context of sound economic development, the advantages of multiparty information resource sharing and acquisition have been highlighted, and people's living standards have been greatly improved, which also puts forward higher demands for the sports industry. It has become an important force to promote the sustainable development of economy and society [3]. The General Administration of Sport of China clearly proposed "Internet + sports" in the Outline of the 13th Five-Year Plan, officially opening the development process of the integration of the Internet and sports. The Opinions on Strengthening the Construction of National Fitness Facilities and Developing Mass Sports issued by the General Office of the State Council in 2020 is proposed to strengthen the construction of national fitness facilities, develop mass sports, promote the construction of fitness facilities, promote the vigorous development of mass sports, and improve the public service 
level of national fitness [4]. In the era of advancing with The Times of Information Technology, sports and the depth of the fusion of the Internet have become a historic turning point in the development of sports industry to encourage the development of the national fitness and sports venue construction process; the development of sports industry and sports information resource sharing becomes hotspot; under the background of big data, the analysis and processing of massive data information are a new challenge for the development of sports industry. The sharing and development of sports information resources based on cloud platform and the analysis and research of sports big data are of great significance.

Liu and $\mathrm{Wu}$ conducted experiments and deployment of the cloud platform for small- and medium-sized enterprises through OpenStack open source cloud framework in order to solve the construction needs of the information service platform for small- and medium-sized enterprises and get rid of the problems of decentralized management and low resource utilization of traditional enterprise information system in the study of the service cloud platform architecture for small- and medium-sized enterprises based on OpenStack. The overall framework of the service platform for small- and medium-sized enterprises is proposed, and the research results show that OpenStack is a private cloud platform more suitable for the construction of small- and medium-sized enterprises [5]. Yi et al. applied the deployment and management of OpenStack cloud platform in the course teaching reform and practice. OpenStack deployment is mainly based on private cloud computing technology, and computing, storage, and network resources are provided to users through the deployment of OpenStack cloud computing environment [6]. Tang and Huang, in the study of high availability of OpenStack-based cloud platform, analyzed high availability cluster and load balancing technology, designed Pacemaker + HAProxy technical solution, and deployed high availability of OpenStack's core component database and mirror service. The high availability of OpenStack cloud platform has been realized [7]. Zhong proposed in his study that OpenStack cloud experiment platform has a high utility ratio and a low expansion cost, which can effectively improve the efficiency of teaching and scientific research experiments. The architecture of OpenStack platform includes user interface, various services, hardware resources, and monitoring and configuration management tools. It has great potential in distributed computing, virtual desktop, and other applications and can provide flexible teaching and research experimental computing services for universities and enterprises [8]. Wang and $\mathrm{Wu}$ studied the construction of the innovation and entrepreneurship cloud platform for local universities based on OpenStack. Starting from the construction status of the innovation and entrepreneurship platform for local universities, they adopted the open source KVM technology to realize computing virtualization and used the storage capacity of Ceph integrated server to realize storage virtualization, thus providing various kinds of virtual innovation and entrepreneurship practice resources. Compared with traditional innovation and entrepreneurship platforms, the innovation and entrepreneurship cloud platform has the characteristics of easy expansion, high resource integration, and low construction cost, which can effectively meet the different demands of local universities for innovation and entrepreneurship resources [9]. Wen et al. analyzed the technical principles of cloud computing and open source cloud platform OpenStack in their research. First, they design and implement a platform that can virtualize and centralize the network with the method of layered design by referring to the traditional network management mode and scheme and test it. Secondly, the platform can quickly deploy and manage the virtual network and create virtual machines according to the needs of users, so as to realize the allocation of resources on demand and the efficient rate [10]. Xin et al. studied the Glance service in OpenStack platform and put forward the position of Glance service in OpenStack platform and the relationship between it and other services and proposed an OpenStack-based private cloud construction scheme suitable for small- and medium-sized enterprises [11]. Yao and Yan combined with a variety of load balancing, IP drift, network routing redundancy protocol (VRRP), database cluster (Galera), distributed cluster (Cech), and other technologies in the research of OpenStack high availability distributed storage scheme design and implementation. This paper proposes a method that can realize the high availability of distributed platform storage scheme by means of business load balancing, service instance failover, and other methods in the case of single node failure, network congestion, and high data outbreak [12].

The innovations of this paper are as follows: (1) sports information resource sharing under the OpenStack cloud computing platform is proposed; (2) empirical analysis of sports industry big data from the perspective of information resource sharing is performed; (3) the healthy development of the information resources of the sports cloud platform is proposed.

The disadvantages of this paper are as follows: (1) research orientation: this study focuses on the data analysis of sports industry; (2) limitations of the study: this paper briefly explains the OpenStack cloud platform, but how to implement the analysis is not the focus of this study. Therefore, it does not give a detailed explanation of how to implement the analysis.

\section{Research Method of Big Data in Sports Industry Based on OpenStack Cloud Platform}

\subsection{Cloud Platform Analysis of OpenStack}

2.1.1. Overview of OpenStack. OpenStack is a free software and open source project developed by NASA and Rackspace under the Apache license to provide software for the construction and management of public and private clouds. OpenStack is a simple, massively scalable, and content-rich cloud computing operating system that supports almost all types of cloud environments. At the same time, OpenStack also provides framework standards and APIs, and service calls between all modules of OpenStack are realized through standard virtualized APIs. OpenStack is written with high- 
quality Python code, and the quality problems and security holes of the open source code are easier to be found and corrected [13]. When establishing the OpenStack system, it mainly includes core service components such as Keystone, Glance, Nova, Neutron, Horizon, and Swift.

Keystone is an authentication service that provides authentication and authorization services for other OpenStack services and an endpoint directory for all OpenStack services. As an OpenStack identity authentication module, Keystone provides a unified identity authentication service for OpenStack service and users, mainly responsible for authentication, service rules, and service instructions [14].

Glance is a mirroring service for storing and retrieving disk images of virtual machines, which OpenStack computing uses when the instance is deployed. Glance is an important mirror query, management, registration, and transport component, which needs to be used for cloud host creation, startup, snapshot, and other services [11].

Nova is a compute service for lifecycle management of compute instances in the OpenStack environment. On-demand response includes operations such as generation, scheduling, and recycling of virtual machines. Nova service, as the core module of the whole system, completes the management and control capabilities related to virtual machine instance lifecycle management, cloud platform network management, and cloud platform storage management [15].

Neutron ensures network services that provide network connectivity to other OpenStack services. In OpenStack calculation, API is provided for users to define the network and use it. Neutron based on plug-in architecture can support numerous network providers and technologies. Neutron networks can realize the flexible division of physical networks and provide each user with an independent network environment in a multiuser and multitenant environment [16].

Horizon provides a web-based management portal that interacts with OpenStack's underlying services to allocate IP addresses and configure access control and plays the role of a dashboard in self-service and management activities. Through the web interface, users can obtain resources of the platform and simplify user operations $[17,18]$.

Swift's HTTP-based application interface stores and arbitrarily retrieves unstructured data objects. With data replication and extensible architecture, you can store written objects and files to multiple hard disks [19]. As a service management module of computer, SWIFT has the function of using and deploying computer information resources and realizes the control of virtual server and system [20].

2.1.2. OpenStack System Architecture. From the functional point of view of OpenStack system, all the constituent subsystems and services of the project jointly provide IaaS services, and the subsystems and services can integrate and call each other through standardized public service interface API. Each system can be independently deployed and used, and the system is internally divided into three levels: API, logical processing (Manager), and underlying Driver adaptation [21].
The management of OpenStack cloud computing management platform includes visual project management of Dashboard, command-line management of Client Console, and management of technical developers of RESTful API [22].

The three-tier service system of cloud computing includes infrastructure-as-a-service, platform-as-a-service, and software-as-a-service. Cloud computing can centralize and share resources such as servers, networks, storage, and application software and provide them to computers, other devices, or end users on demand [23].

According to user needs, OpenStack service components can be selectively installed into multiple host nodes. The method of multihost nodes is also known as multinode deployment. To put it simply, different hosts install different service components to assume different service roles. The platform of multinode deployment has the characteristics of easy expansion, rich functions, and strong maneuverability.

2.1.3. OpenStack Deployment Architecture. The common deployment methods of OpenStack's multinode deployment platform include two-node deployment architecture and three-node deployment architecture. In a two-node architecture, the configuration node consists of a control node and a compute node. In a three-node architecture, the configuration node consists of control node, network node, and compute node. There is only one control node and it can control all services including Keystone, Glance, Nova, Neutron, and API services, MySQL database, and message system [24].

Yang proposed that OpenStack provides an open source software framework for the construction and management of cloud computing. By building a private teaching cloud in schools, software and hardware resources can be shared, jointly built, and integrated, so as to improve teaching quality, service level, and management efficiency [25].

$\mathrm{Wu}$ et al. proposed two practical deployment modes of OpenStack nodes, including master control node and computing node. This deployment mode is characterized by (1) easy expansion: the practical application may be extended due to the expansion of the business, and whether the deployment is simple and convenient affects the difficulty of the expansion; (2) easy maintenance: when the order of magnitude of the node increases, the difficulty of maintenance needs to be considered; (3) high stability: whether the architecture has high reliability and can provide services continuously and stably [24].

$\mathrm{Li}$ et al. proposed an OpenStack-based private cloud platform architecture scheme in their research on the design of OpenStack-based private cloud platform and believed that PackStack could quickly deploy a set of multinode OpenStack clusters on CentOS. It is characterized by simple operation and rapid deployment [26].

\subsection{Research Methods of Big Data in Sports Industry}

2.2.1. Index Selection. The layout and structure of the industry are controlled by certain economic and technological conditions. In order to promote the coordinated development of the 
social economy, it is necessary to adjust the industrial structure appropriately, improve the conversion ability of the industrial structure, and promote the development of the industrial structure to the direction of rationalization and advancement [27]. In this paper, contribution rate and pull rate are selected as indicators to study the development of the sports industry. By analyzing the total volume and structural characteristics of the sports industry, the influence relationship of the sports industry in the process of economic development is studied.

The contribution rate is the ratio of the increment of a part of the population to the increment of the population. In order to study the contribution rate of sports industry development to economic development, since the added value of sports industry is a part of regional GDP, the contribution rate can be represented by the ratio of the incremental value of sports industry to the incremental value of GDP [28]. The calculation formula is as follows:

$$
\mathrm{SR}_{t}=\frac{Z_{t}-Z_{t-1}}{\mathrm{GDP}_{t}-\mathrm{GDP}_{t-1}} \times 100 \% .
$$

In the formula, $t$ is the time, $\mathrm{SR}_{t}$ is the contribution rate of sports industry, $Z_{t}$ is the total revenue of the sports industry in the current year, and $\mathrm{GDP}_{t}$ is the gross domestic product of the year.

Pull rate refers to the percentage of total growth driven by the growth of a certain factor [29]. In order to study the driving rate of sports industry for economic development, the calculation formula is as follows:

$$
\mathrm{SP}_{t}=\mathrm{SR}_{t} \times \mathrm{GP}_{t} \times 100 \% .
$$

In the formula, $t$ is the time, $\mathrm{SP}_{t}$ is the driving rate of sports industry, and $\mathrm{GP}_{t}$ is the GDP growth rate.

2.2.2. Data Source of Sports Industry. Data demand is the added value data of the economic development of the national sports industry, the relevant information of the economic development data of the national sports industry, and the statistical classification standard of the sports industry.

Data sources: data of the added value of the national sports industry from 2015 to 2019 are from Announcement of the Data on the Scale and the added value of the national sports industry jointly issued by the National Bureau of Statistics of the General Administration of Sport, and data of the national economic development from 2015 to 2019 are from Statistical Bulletin of National Economic and Social Development of the National Bureau of Statistics. Sports Industry Statistical Classification (2019) is adopted for the accounting classification of sports industry. Based on the Classification of Industries in the National Economy (GB/T 4754-2017), it is a reclassification of related activities conforming to the characteristics of sports industry in the classification of industries in the national economy [30].

Data analysis software requirements: Excel 2016 and SPSS 24.0.

2.2.3. Data Collection and Processing. The research takes the national sports industry economic index as the research object.
According to the Statistical Classification of Sports Industry, the sports industry is divided into 11 categories. It mainly includes sports management activities, sports competition performances, sports fitness and leisure activities, sports venue services, sports intermediary services, sports education and training, sports media and information services, sports goods and related product agents, other sports services, sports goods and related product manufacturing, and sports facilities. The added value data of the national sports industry include the added value data and the proportion of each year according to the statistical classification standard of the sports industry. The national economic development data include economic indicators and years such as GDP, the number of employed people, social fixed asset investment, and per capita GDP. The data are summarized and sorted into Excel 2016 software, and descriptive statistical analysis is conducted on the collected data. In order to verify the appropriateness and reliability of the measured variables, exploratory factor analysis and confirmatory factor analysis were performed on standardized data using SPSS 24.0.

2.3. Research Ideas. This paper takes the information resource sharing and sports industry big data research under the background of OpenStack cloud platform as the theme, collects data, collates data, and determines research methods. The research contents include (1) information resource sharing of sports industry based on OpenStack platform, which mainly realizes resource sharing from the aspects of OpenStack system and node deployment; (2) big data analysis of sports industry, mainly analyzing the influence of sports industry on economic development from two aspects: contribution rate and pull rate. The specific process is shown in Figure 1.

\section{Research on Information Resource Sharing of Sports Industry Based on OpenStack Cloud Platform}

\subsection{Design of Sports Information Resource Sharing Module}

3.1.1. Asynchronous Deployment of Information Resource Sharing. As the control part of cloud organization, Nova component in OpenStack realizes the configuration of resources through virtual machine, which can manage the network and control the user's access to the cloud. The information resources of Nova service are very complex. During the deployment process, API node deployment and compute node deployment are the main ones. The deployment process of Nova service is as follows: the Nova-API receives requests from the client and the creation of the virtual machine, validates them, and forwards them to the Nova-Scheduler, who forwards the creation of the virtual machine to the NovaCompute service on the selected node. Nova-The Compute service runs to create virtual machines on compute nodes, and Nova-Compute forwards messages to libvirt, which in turn hands off tasks to KVM and Xen.

It provides API services for users to implement initial deployment and virtual interaction functions and integrates 


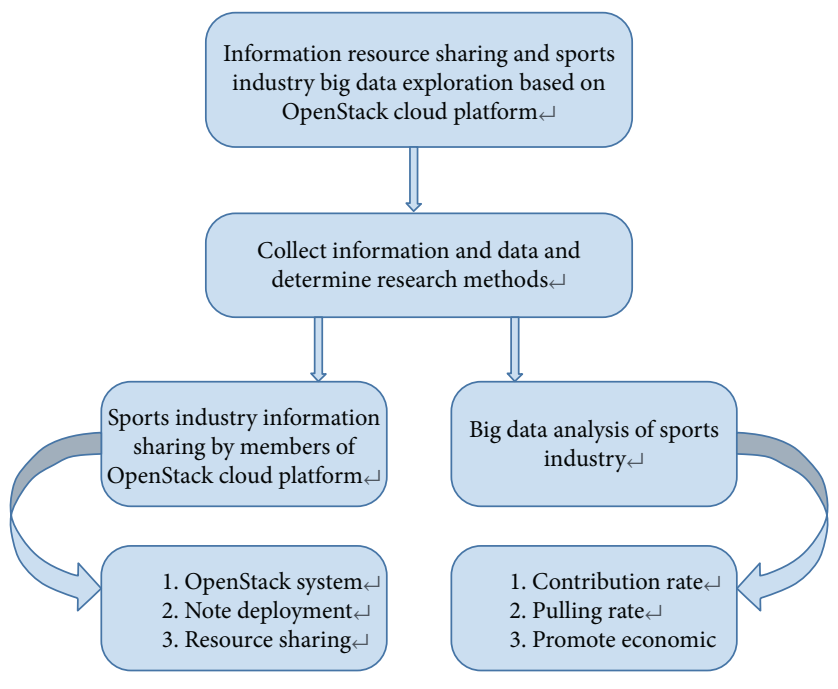

FIgURE 1: The research process.

sports information resources through database services and virtual network deployment. The tasks are simplified through database and virtual network services, and asynchronous deployment of message passing and information sharing tasks is realized.

3.1.2. Storage Service Deployment. In order to prevent data loss and data storage capacity problems, the durability and scalability of data can be improved through the SWIFT cloud storage service component. Even in a small deployment environment, high data persistence can be maintained in the Swift cloud storage service to prevent data loss. At the same time, Swift cloud storage can increase the storage capacity, improve the overall performance of the system, and increase the user experience. In the deployment process, in order to increase the security and stability of the system, when the geographical location is different, according to different needs, different storage nodes are allocated to different areas; even if there are multiple storage nodes, you can also ensure the acquisition of effective data.

In a fast access system, a sudden network outage may result in data, but delayed update operation will be added to the update list again. This makes up for the previous operation and allows the system to effectively pass the operation instructions.

3.1.3. Network Service Creation. OpenStack provides virtual network by Neutron, which can realize the communication between virtual machines and physical machines and provide virtual switching services and DHCP services for virtual machines. In order to enable virtual machines to access the external network and provide NAT services, it can be called by other components of OpenStack during the service process. The establishment process of the node-side network service is as follows: create Neutron service, create network service API Endpoint, install the provider network component, edit relevant files, make configuration modification, configure Linux bridge agent, configure DHCP agent, configure metadata agent, create file link, synchronize database, and restart computing service. The process of creating a compute node-side network service is as follows: install the compute node-side network component, make configuration changes, configure the Linux bridge agent, restart the compute service, and start the Linux bridge agent.

The sports big data information resources are connected to the data network, and the big data of sports information resources are turned into virtual data in cloud deployment, so as to truly realize the sports big data network system. In the network connection, both external networks and public networks can be accessed, and sports big data information resources can be shared anytime and anywhere to achieve undifferentiated network access, which is more conducive to data transmission and sharing.

\subsection{OpenStack Service Inspection and Troubleshooting}

3.2.1. Service Inspection. Invoke the Mova-Manage tool to verify that the OpenStack computing service is running properly, understand the state of the environment through different parameters, and detect the different components of the computing service.

Check the OpenStack web service. On the control node, execute the netstat-anlp 1 grep 9696 command to check whether the network Server API service is running on TCP port 9696.

Check the OpenStack object storage service. Swift, the OpenStack object storage service, has some built-in tools that check the health of the service and execute commands on the Swift node.

3.2.2. Troubleshooting Storage Services. As a storage construction system with high reliability, Swift is able to deal with most of the failure problems in the system. The client test of Swift failure operation is eliminated, and only the log operation is considered. First, there may be identity authentication problems, which are mainly caused by 
certificate errors in user or system configuration. A Swift system that supports OpenStack's authentication service requires you to manually set the authentication steps. Second, if a disk in the Swift storage environment fails, verify that the disk is unmounted, avoid Swift writing data, replace the disk, and readjust commands. Third, if the server is not running for a few hours, Swift will still work, and if it lasts longer, the server will need to be removed from the command.

\section{Big Data Analysis of Sports Industry}

\subsection{Economic Development Status of Sports Industry}

4.1.1. The Current Situation of Sports Industry Development. In order to study the impact of the sports industry on economic development, the sports industry accounting classification adopts the Sports Industry Statistical Classification (2019). Based on the Classification of Industries in National Economy (GB/T 4754-2017), the added value and proportion of 11 sports industry categories are sorted out and counted in the table. It can be seen from the data that in the category of sports industry from 2015 to 2019, the manufacturing of sporting goods and related products has the largest added value and proportion, and the second is the agency of sporting goods and related products. At the same time, the manufacturing of sporting goods and related products and the agency of sporting goods and related products account for the highest proportion of $78.6 \%$ in that year. The added value of the sports industry reached 431.79 billion yuan. Specific data are shown in Tables 1 and 2 and Figure 2.

Sports venue services ranked third from 2015 to 2017, with an added value of 45.81 billion yuan in 2015 , accounting for $8.3 \%$. In 2016, the added value was 56.76 billion yuan, accounting for $8.8 \%$. In 2017 , the added value was 67.82 billion yuan, accounting for 8.7 percent. Rounding out the top three in 2018-2019 was physical education and training, with an added value of 142.5 billion yuan in 2018, accounting for 14.1 percent. In 2019, the added value was 152.49 billion yuan, accounting for 13.6 percent. In 2018 and 2019, the added value of sports venue services was slightly lower than that of physical education and training. In 2018, the added value of sports venue services was 85.5 billion yuan, accounting for $8.5 \%$, and in 2019 , the added value of sports venue services was 101.22 billion yuan, accounting for $9.0 \%$.

In 2018, the added value of other sports services was 61.48 billion yuan, accounting for $6.1 \%$ of the total. In 2019 , the added value of sports, fitness, and leisure activities is 83.19 billion yuan, accounting for $7.4 \%$, while the added value of other sports services is 70.07 billion yuan, accounting for $6.3 \%$, which is slightly lower than that of sports, fitness, and leisure activities. The total added value of the sports industry was 549.44 billion yuan in 2015, 647.48 billion yuan in 2016, 781.14 billion yuan in 2017 , $1,007.8$ billion yuan in 2018, and 1,124 .81 billion yuan in 2019. The added value of sports industry is increasing year by year.
4.1.2. The Current Economic Situation Related to the Development of Sports Industry. Indicators related to the development of the sports industry include the GDP, the number of employed people, the total investment in fixed assets of the whole society, and the per capita GDP. From 2015 to 2019, China's GDP and per capita GDP showed an upward trend year by year, reaching 67,6708 trillion yuan in $2015,74,4127$ trillion yuan in $2016,8,27122$ trillion yuan in 2017, and 9,0309 trillion yuan in 2018. China's GDP in 2019 is 9.9086 .5 trillion yuan. In 2015, the per capita GDP was 4.9355 .1 trillion yuan. From 2015 to 2019 , the per capita GDP showed an upward trend year by year, reaching 7.089.2 trillion yuan in 2019. From 2015 to 2018, the investment in fixed assets of the whole society showed an upward trend. In 2019, the investment in fixed assets of the whole society declined slightly. In 2015, the investment in fixed assets of the whole society was 5.62 trillion yuan, in 2016, it was 6.06466 trillion yuan, and in 2017, it was 6.41238 trillion yuan. In 2018, the total fixed asset investment reached the highest value of 6.4567 .5 trillion yuan, and in 2019, the total fixed asset investment dropped to 5.6087.4 trillion yuan. There was no significant change in the number of employed persons from 2015 to 2019. In 2017, the highest number of employed persons was 776.4 million. In 2015 to 2017, the number of employed persons increased slightly, and in 2018 to 2019 , the number of employed persons decreased slightly. Specific data are shown in Table 3 and Figure 3.

\subsection{Influence of Sports Industry on Economic Development}

\subsubsection{The Contribution of Sports Industry to Economic} Development. The contribution rate of sports industry reflects the contribution of sports industry development to economic development. Can be seen from the collating of data, the "sports fitness and leisure activities, stadium services, physical education and training, sporting goods and related products agents, other sports services, sporting goods and related products manufacturing" promote larger contribution to the development of the economy, the following only for more than six industry has contributed to a detailed description. The detailed content of the contribution rate of sports industry is shown in Table 4 .

In the category of sports industry, the top three contribution rates to economic development are sports goods and related products manufacturing, sports goods and related products agent, and physical education and training. Overall, the contribution rate of the manufacturing of sporting goods and related products showed a downward trend from 2015 to 2019, but the contribution rate increased slightly in 2018. The contribution rate of the manufacturing of sporting goods and related products was the highest $6.85 \%$ in 2015 , followed by the contribution rate of $4.64 \%$ in 2018 and $4.25 \%$ in 2016 . From 2015 to 2019 , the contribution rate of sports goods and related products agency showed a downward trend. The contribution rate of sports goods and related products agency in 2015 was the highest $3.88 \%$, and the difference between the contribution rate of sports goods and related products agency in 2016 to 2018 was small, and 
TABLE 1: Economic added value of sports industry.

\begin{tabular}{|c|c|c|c|c|c|c|}
\hline & Classification of sports industry & 2019 & 2018 & 2017 & 2016 & 2015 \\
\hline 1 & Sports management activities & 451.9 & 390 & 262.6 & 143.8 & 115 \\
\hline 2 & Sports competition performance activities & 122.3 & 103 & 91.2 & 65.5 & 52.6 \\
\hline 3 & Sports fitness and leisure activities & 831.9 & 477 & 254.9 & 172.9 & 129.4 \\
\hline 4 & Stadium services & 1012.2 & 855 & 678.2 & 567.6 & 458.1 \\
\hline 5 & Sports intermediary service & 117.8 & 106 & 24.6 & 17.8 & 14.0 \\
\hline 6 & Physical education and training & 1524.9 & 1425 & 266.5 & 230.6 & 191.8 \\
\hline 7 & Sports media and information services & 285.1 & 230 & 57.7 & 44.1 & 40.8 \\
\hline 8 & Sporting goods and related products agent & 2562 & 2328 & 2615.8 & 2138.7 & 1562.4 \\
\hline 9 & Other sports services & 707 & 614.8 & 197.2 & 179.7 & 139.6 \\
\hline 10 & Sporting goods and related products manufacturing & 3421 & 3396.3 & 3264.6 & 2863.9 & 2755.5 \\
\hline \multirow[t]{2}{*}{11} & Construction of sports facilities & 211.9 & 151.2 & 97.8 & 50.3 & 35.3 \\
\hline & Total & 11248.1 & 10078 & 7811.4 & 6474.8 & 5494.4 \\
\hline
\end{tabular}

Source: National Bureau of Statistics (National Bureau of Statistics of General Administration of Sport of China Joint Announcement on the Data of Scale and Value Added of National Sports Industry from 2015 to 2019); unit: 100 million yuan.

TABLE 2: Economic added value ratio of sports industry.

\begin{tabular}{|c|c|c|c|c|c|c|}
\hline & Classification of sports industry & 2019 & 2018 & 2017 & 2016 & 2015 \\
\hline 1 & Sports management activities & 4.0 & 3.9 & 3.4 & 2.2 & 2.1 \\
\hline 2 & Sports competition performance activities & 1.1 & 1.0 & 1.2 & 1.0 & 1.0 \\
\hline 3 & Sports fitness and leisure activities & 7.4 & 4.7 & 3.3 & 2.7 & 2.4 \\
\hline 4 & Stadium services & 9.0 & 8.5 & 8.7 & 8.8 & 8.3 \\
\hline 5 & Sports intermediary service & 1.0 & 1.1 & 0.3 & 0.3 & 0.3 \\
\hline 6 & Physical education and training & 13.6 & 14.1 & 3.4 & 3.6 & 3.5 \\
\hline 7 & Sports media and information services & 2.5 & 2.3 & 0.7 & 0.7 & 0.7 \\
\hline 8 & Sporting goods and related products agent & 22.8 & 23.1 & 33.5 & 33.0 & 28.4 \\
\hline 9 & Other sports services & 6.3 & 6.1 & 2.5 & 2.8 & 2.5 \\
\hline 10 & Sporting goods and related products manufacturing & 30.4 & 33.7 & 41.8 & 44.2 & 50.2 \\
\hline \multirow[t]{2}{*}{11} & Construction of sports facilities & 1.9 & 1.5 & 1.3 & 0.8 & 0.6 \\
\hline & Total & 100.0 & 100.0 & 100.0 & 100.0 & 100.0 \\
\hline
\end{tabular}

Unit: \%.

the lowest contribution rate in 2019 was $2.83 \%$. The contribution rate of physical education and training was 1.95 percent in 2018 and 1.68 percent in 2019 . There was a small difference in the contribution rate of physical education and training from 2015 to 2017. The lowest contribution rate of physical education and training in 2017 was 0.32 percent. See Figure 4 for details.

Stadium services, sports fitness and leisure activities, and other sports services also contribute significantly to economic development. From the perspective of stadium services, the contribution rate of stadium services in 2018 was the highest $1.17 \%, 2015$ was $1.14 \%, 2019$ was $1.12 \%$, and 2016 and 2017 were $0.84 \%$ and $0.82 \%$, respectively. From the perspective of physical fitness and leisure activities, the contribution rate of physical fitness and leisure activities showed an increasing trend from 2016 to 2019. The lowest contribution rate of physical fitness and leisure activities was $0.26 \%$ in 2016 , the highest was $0.92 \%$ in 2019 , and the contribution rate of physical fitness and leisure activities in 2015 was $0.32 \%$. From the perspective of other sports services, the contribution rate of other sports services showed a downward trend from 2015 to 2017. In 2018, the contribution rate of other sports services rose to the highest value of $0.84 \%$. In 2019 , the contribution rate of other sports services decreased slightly compared with 2018, with $0.78 \%$ in 2019 and $0.24 \%$ in 2017. See Figure 5 for details.

\subsubsection{The Driving Effect of Sports Industry on Economic} Development. The driving rate of sports industry reflects the driving effect of sports industry on economic development. From the data, it can be seen that sports products and related products manufacturing, sports goods and related products agency, physical education and training, and sports venue services have a high pull rate index. The following is a specific description of the pull rate of the above four industries. The detailed content of sports industry pulling rate is shown in Table 5 .

The manufacturing of sports products and related products has the most obvious driving effect on economic development. From 2015 to 2017, the driving effect of the manufacturing of sports products and related products is on the rise. From 2017 to 2019, the driving effect of the manufacturing of sports products and related products is decreasing year by year, but the overall change range is small. The highest pull rate was $0.432 \%$ in 2017 , and the lowest was $0.378 \%$ in 2019 . The driving rate of sports goods and related products agent increased year by year from 2015 to 2017, the 


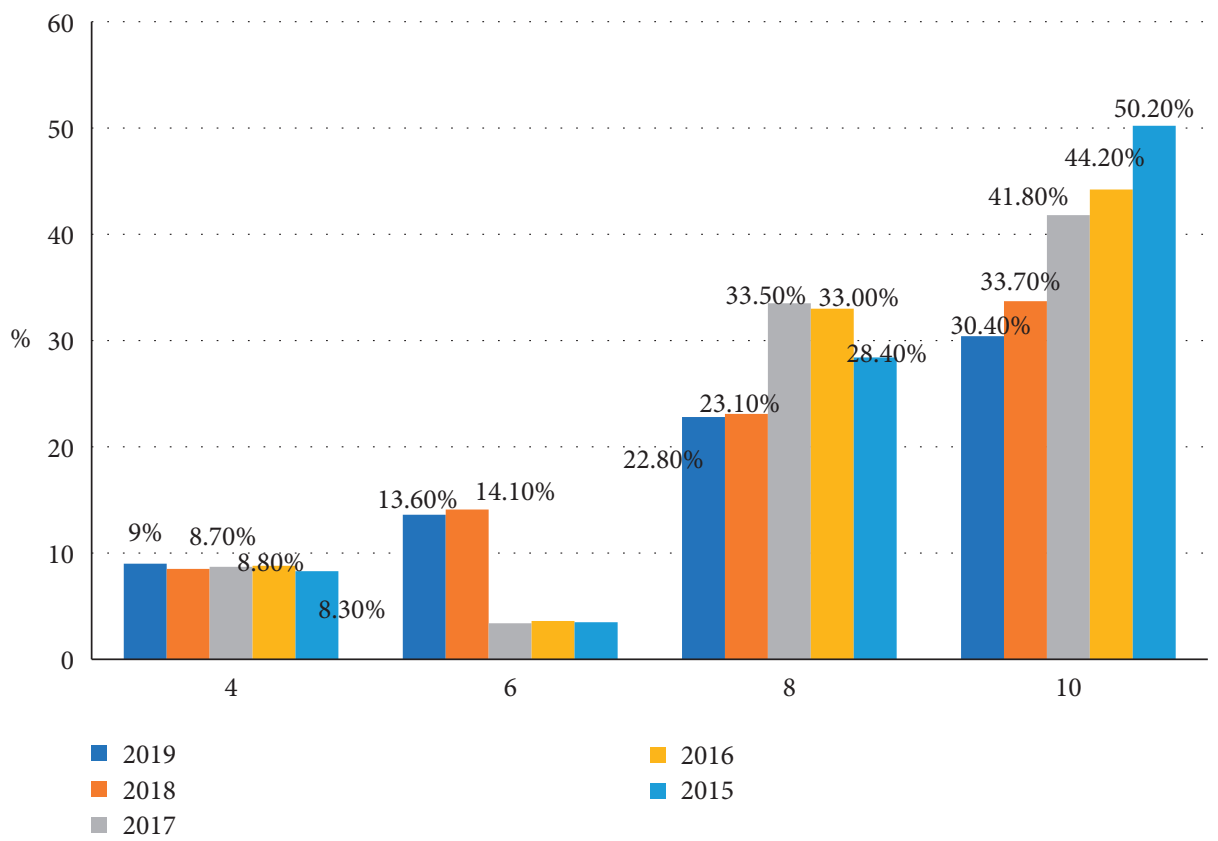

FIgURE 2: Economic added value of sports industry.

TABle 3: National economic development data.

\begin{tabular}{|c|c|c|c|c|c|c|}
\hline & Economic indicators & 2019 & 2018 & 2017 & 2016 & 2015 \\
\hline 1 & Gross domestic product & 990865 & 900309 & 827122 & 744127 & 676708 \\
\hline 2 & Number of employed persons & 77471 & 77586 & 77640 & 77603 & 77451 \\
\hline 3 & Investment in fixed assets throughout the country & 560874 & 645675 & 641238 & 606466 & 562000 \\
\hline 4 & Per capita GDP & 70892 & 64644 & 59660 & 53980 & 49351 \\
\hline
\end{tabular}

Source: National Bureau of Statistics (2015-2019 Statistical Bulletin on National Economic and Social Development); unit: 100 million yuan, ten thousand people.

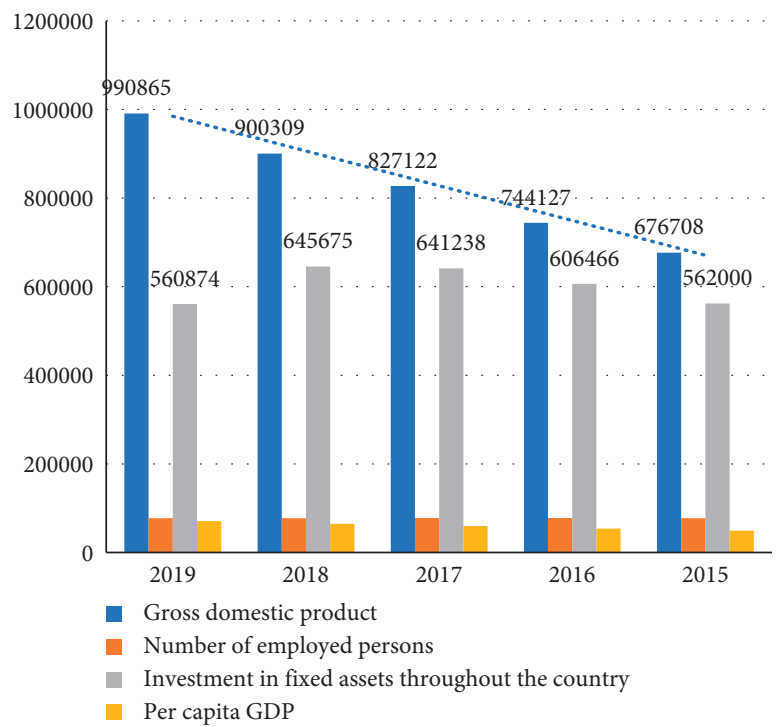

Figure 3: National economic development data.

driving rate reached the maximum of $0.347 \%$ in 2017 and the lowest of $0.233 \%$ in 2015 . The driving rate of sports goods and related products agent was $0.283 \%$ in 2018 and 2019 , and the driving rate was reduced compared with that of 2018 and 2017. From 2015 to 2019, the pull rate of physical education and training showed an upward trend, while from 2015 to 
TABLE 4: Contribution rate of sports industry.

\begin{tabular}{|c|c|c|c|c|c|c|}
\hline & \multirow{2}{*}{ Classification of sports industry } & \multicolumn{5}{|c|}{$\mathrm{SR}_{t}(\%)$} \\
\hline & & 2019 & 2018 & 2017 & 2016 & 2015 \\
\hline 1 & Sports management activities & 0.50 & 0.53 & 0.32 & 0.21 & 0.28 \\
\hline 2 & Sports competition performance activities & 0.14 & 0.14 & 0.11 & 0.10 & 0.13 \\
\hline 3 & Sports fitness and leisure activities & 0.92 & 0.65 & 0.31 & 0.26 & 0.32 \\
\hline 4 & Stadium services & 1.12 & 1.17 & 0.82 & 0.84 & 1.14 \\
\hline 5 & Sports intermediary service & 0.13 & 0.14 & 0.03 & 0.03 & 0.03 \\
\hline 6 & Physical education and training & 1.68 & 1.95 & 0.32 & 0.34 & 0.48 \\
\hline 7 & Sports media and information services & 0.31 & 0.31 & 0.07 & 0.07 & 0.10 \\
\hline 8 & Sporting goods and related products agent & 2.83 & 3.18 & 3.15 & 3.17 & 3.88 \\
\hline 9 & Other sports services & 0.78 & 0.84 & 0.24 & 0.27 & 0.35 \\
\hline 10 & Sporting goods and related products manufacturing & 3.78 & 4.64 & 3.93 & 4.25 & 6.85 \\
\hline 11 & Construction of sports facilities & 0.23 & 0.21 & 0.12 & 0.07 & 0.09 \\
\hline & Total & 12.42 & 13.77 & 9.41 & 9.60 & 13.65 \\
\hline
\end{tabular}

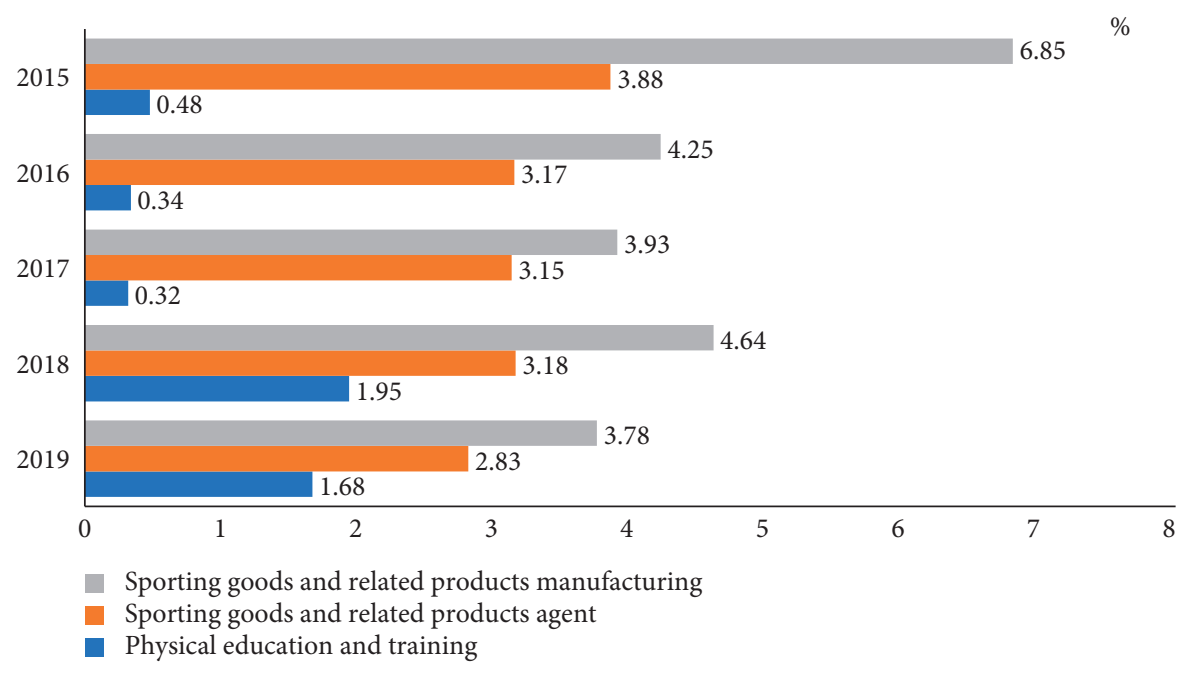

FIgURE 4: Contribution rate of sports industry.

2017, the rise rate was slow. The pull rate increased significantly in 2018 and 2019, with the lowest value of $0.029 \%$ in 2015 and the highest value of $0.168 \%$ in 2019 . The pull rate of stadium services increased year by year from 2015 to 2019. The lowest pull rate of stadium services in 2015 was $0.068 \%$, and the highest one in 2019 was $0.112 \%$. See Figure 6 for details.

\subsection{Contribution and Driving Effect of Sports Industry to} Economy. Figure 7 shows the influence relationship between the contribution rate and pulling rate of sports industry and GDP growth rate. It can be seen from the figure that the contribution rate of sports industry to economic development was $13.65 \%$ in $2015,9.6 \%$ in 2016 , $9.41 \%$ in 2017 , and the contribution rate increased significantly in 2018. The overall contribution rate of the sports industry reached $13.77 \%$, and the contribution rate of the sports industry in 2019 was $12.42 \%$. From the perspective of the driving effect of sports industry on economic development, from 2015 to 2019, the driving effect of sports industry on economic development is not very obvious, with the driving rate of $0.819 \%$ in 2015 , $0.96 \%$ in 2016 , and $1.035 \%$ in 2017 . The pull rate in 2018 is $1.226 \%$, and the pull rate in 2019 is $1.242 \%$. The growth rate of GDP began to increase year by year in 2015 and reached a maximum of $11.15 \%$ in 2017. In 2018, the growth rate of GDP decreased, and in 2019, the growth rate of GDP rose to $10.05 \%$. On the whole, the average contribution rate of the sports industry is $11.77 \%$, and the average pull rate of the sports industry is $1.056 \%$. The values of the contribution rate and pull rate of the sports industry are both positive. The sports industry has obvious contribution and pull effect on the economic development, so the development of the sports industry promotes the development of the social economy. It has a positive influence on economic development and thus promotes economic development. See Figure 7 for details. 


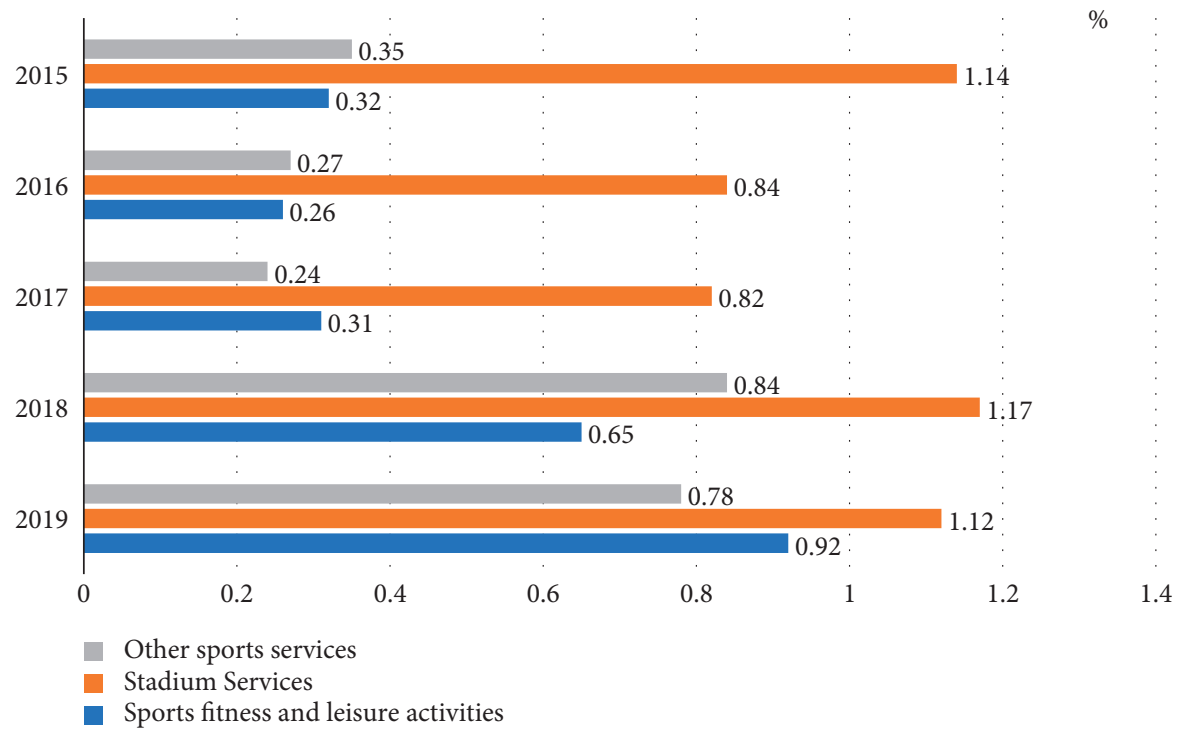

FIGURE 5: Contribution rate of sports industry.

Table 5: The pull rate of sports industry.

\begin{tabular}{|c|c|c|c|c|c|c|}
\hline & \multirow{2}{*}{ Classification of sports industry } & \multicolumn{5}{|c|}{$\mathrm{SP}_{t}(\%)$} \\
\hline & & 2019 & 2018 & 2017 & 2016 & 2015 \\
\hline 1 & Sports management activities & 0.050 & 0.047 & 0.035 & 0.021 & 0.017 \\
\hline 2 & Sports competition performance activities & 0.014 & 0.012 & 0.012 & 0.010 & 0.008 \\
\hline 3 & Sports fitness and leisure activities & 0.092 & 0.058 & 0.034 & 0.026 & 0.019 \\
\hline 4 & Stadium services & 0.112 & 0.104 & 0.090 & 0.084 & 0.068 \\
\hline 5 & Sports intermediary service & 0.013 & 0.012 & 0.003 & 0.003 & 0.002 \\
\hline 6 & Physical education and training & 0.168 & 0.174 & 0.035 & 0.034 & 0.029 \\
\hline 7 & Sports media and information services & 0.031 & 0.028 & 0.008 & 0.007 & 0.006 \\
\hline 8 & Sporting goods and related products agent & 0.283 & 0.283 & 0.347 & 0.317 & 0.233 \\
\hline 9 & Other sports services & 0.078 & 0.075 & 0.026 & 0.027 & 0.012 \\
\hline 10 & Sporting goods and related products manufacturing & 0.378 & 0.413 & 0.432 & 0.425 & 0.411 \\
\hline 11 & Construction of sports facilities & 0.023 & 0.019 & 0.013 & 0.007 & 0.005 \\
\hline & Total & 1.242 & 1.226 & 1.035 & 0.960 & 0.819 \\
\hline
\end{tabular}

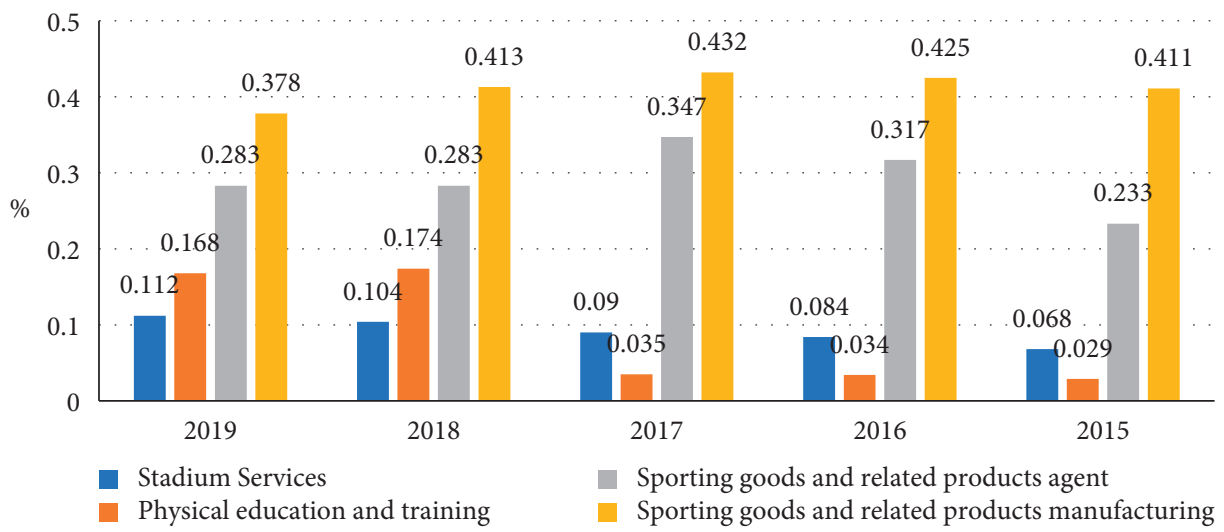

Figure 6: The pull rate of sports industry. 


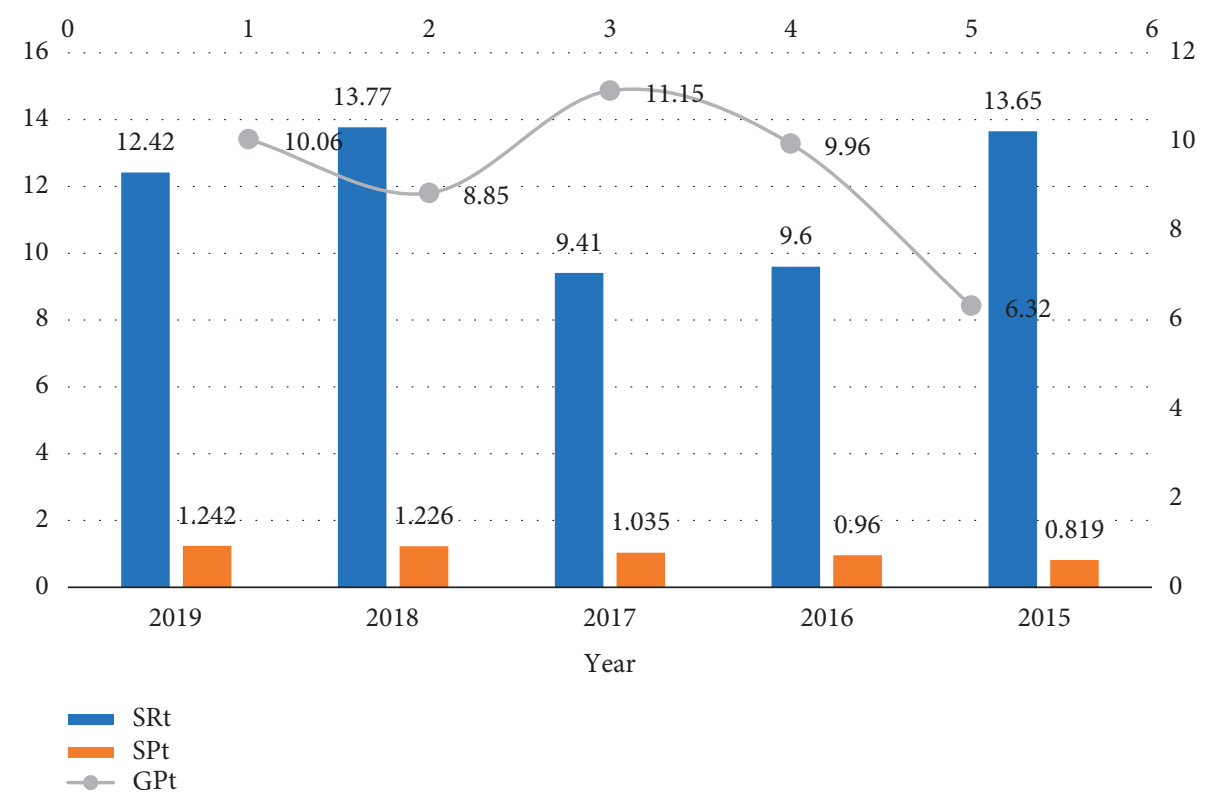

Figure 7: Contribution rate and pull rate of sports industry.

\section{Conclusions}

Statistical analysis according to the scale of the sports industry, sports products and related products manufacturing industry, sports products and related products agents, and physical education and training industry scale continues to expand, but has been to the direction of sports services, sports industry structure also tends to rationalization. In the development process of big data, sports industry is integrated into big data analysis to make data acquisition more intuitive and data analysis more comprehensive, grasp the development trend of sports industry economy, and predict the future development trend of sports industry, so as to promote economic and social development.

Big data are inseparable from us in modern life. In the cloud age, big data also involve various fields. Through the cloud computing platform technology, a large amount of data can be effectively acquired, stored, managed, and analyzed. The application of big data to the sports industry has the following two impacts on the sports industry. First, through the analysis of big data of the sports industry, we can intuitively understand the impact of the sports industry on economic development, which is of profound significance for promoting economic and social development. Secondly, through the design of the information resource sharing module of the cloud platform, the obstacles to the acquisition of big data resources are greatly reduced, making the acquisition of data resources and the analysis of big data more convenient.

\section{Data Availability}

No data were used to support this study.

\section{Conflicts of Interest}

The authors declare no potential conflicts of interest with respect to the research, authorship, and/or publication of this article.

\section{References}

[1] Smart government big data cloud platform construction scheme [EB/OL], 2018.

[2] The State Council Issued the Action Program to Promote the Development of Big Data [EB/OL], Xinhua News Agency, Beijing, China, 2015.

[3] The State Council "Several Opinions on Accelerating the Development of Sports Industry and Promoting Sports Consumption", State Council, Zhongnanhai, Beijing, China, 2014.

[4] Opinions of General Office of the State Council on Strengthening the Construction of National Fitness Facilities and Developing Mass Sports [EB/OL] No, State Council, Zhongnanhai, Beijing, China, 2020.

[5] G. C. Liu and D. Wu, Journal of Jilin University (Engineering and Technology Edition), vol. 38, no. 6, pp. 709-713, 2020.

[6] H. B. Yi, R. N. Chi, Z. Nie, and X. Huang, "Teaching reform and practice of "OpenStack cloud platform deployment and management" course," Journal of Shenzhen Vocational and Technical College, vol. 19, no. 5, pp. 66-71, 2020.

[7] Z. J. Tang and C. Huang, "Research on high availability of cloud platform based on OpenStack," Information \& Computer (Theoretical Edition), vol. 2019, no. 6, pp. 21-22, 2019.

[8] Y. Zhong, "Application research of OpenStack on cloud experimental platform construction," Electronic Design Engineering, vol. 27, no. 8, pp. 24-28+33, 2019.

[9] Y. Wang and Q. L. Wu, "Construction of the cloud platform for innovation and entrepreneurship of local universities based on OpenStack," Experimental Technology and Management, vol. 36, no. 4, pp. 257-260+277, 2019. 
[10] T. T. Wen, H. Z. Li, and S. F. Li, "Design and implementation of virtual network management platform based on OpenStack," Electronic Manufacturing, vol. 2019, no. 10, pp. 47-49, 2019.

[11] Z. L. Xin, D. M. Liang, R. Ma, M. H. Zou, and J. P. Zhang, "Glance service based on OpenStack platform," Henan Science and Technology, vol. 63, no. 23, pp. 33-35, 2020.

[12] J. Yao and N. Yan, "Design and implementation of OpenStack high availability distributed storage scheme," Computer Technology and Development, vol. 29, no. 2, pp. 35-38, 2019.

[13] Y. Y. Zhu, Cloud Computing Architecture and Application, pp. 7-8, South China University of Technology Press, Guangzhou, China, 2017.

[14] X. L. Tian, Z. Yuan, and N. Zhang, "Security research and improvement of OpenStack authentication backend," Journal of Beijing Institute of Electronic Science and Technology, vol. 24, no. 4, pp. 26-31, 2016.

[15] J. L. Mao, "OpenStack Nova service," Computer Networks, vol. 44, no. 3, pp. 60-63, 2018.

[16] Y. Li, Y. Dong, J. Zhang, D. Liang, and J. Zhang, "Neutron services based on OpenStack platform," Computer Programming Skills and Maintenance, vol. 26, no. 10, pp. 12-13+46, 2020.

[17] Z. Y. Gong, "Implementation of OpenStack Horizon automated testing based on selenium," Foreign Electronic Measurement Technology, vol. 36, no. 5, pp. 45-49, 2017.

[18] C. Cheng, Z. X. Zhu, and X. J. Liang, "Research and application of OpenStack Horizon framework," Electronic Science and Technology, vol. 29, no. 4, pp. 84-87, 2016.

[19] Y. Song, D. Y. Zhou, and W. C. Shi, "A method to enhance the security function of OpenStack Swift cloud storage system," Journal of Jilin University (Engineering and Technology Edition), vol. 51, no. 1, pp. 314-322, 2021.

[20] H. D. Huang, "Application of cloud computing in virtual training teaching platform for network security," Electronic Technology, vol. 49, no. 12, pp. 24-25, 2020.

[21] X. L. Zhang, C. Y. Wen, and Z. Zhang, "Research and implementation of high availability storage scheme based on OpenStack Swift," Journal of Chengdu University of Information Technology, vol. 34, no. 1, pp. 44-48, 2019.

[22] J. T. Yin and T. T. Lu, "Research on mobile application construction of OpenStack cloud computing platform," Journal of Jining Normal University, vol. 42, no. 05, pp. 57-61, 2020.

[23] L. Y. Song, "Research and implementation of university cloud data center based on OpenStack," Journal of Chifeng University (Natural Science Edition), vol. 32, no. 15, pp. 33-35, 2016.

[24] M. L. Wu, T. H. Ren, and Y. B. Li, “Application and research of resource management technology of private cloud platform based on OpenStack," Industrial Technology Innovation, vol. 2, no. 3, pp. 334-341, 2015.

[25] X. F. Yang, "Research and implementation of teaching private cloud based on OpenStack open source cloud platform," Information and Computers, vol. 32, no. 4, pp. 188-190, 2020.

[26] H. Li, H. Zhou, H. Zhang, and B. Feng, "EmuStack: an OpenStack-based DTN network emulation platform," in Proceedings of 2016 International Conference on Networking and Network Applications (NaNA), vol. 35, no. 9, pp. 24-26, Hakodate City, Hokkaido, Japan, July 2016.

[27] X. M. Zhang and J. Wang, Industrial Economics, p. 121, Electronic Science and Technology University Press, Xi 'an, China, 2017.
[28] Y. Zhang and J. Y. Xiong, "On the calculation of "contribution rate" and "pull rate"," China Statistics, vol. 64, no. 9, pp. 38-40, 2017.

[29] Y. Li, P. Y. Zhao, and M. Z. Xi, "An empirical study on the driving effect of tourism on economic growth in Xinzhou City: based on the three indicators of tourism dependence, contribution rate and driving rate," Journal of Mianyang Normal University, vol. 34, no. 6, pp. 44-48, 2015.

[30] National Economy Industry Classification Notes》(revised According to Amendment No.1), National Bureau of Statistics, China, 2017. 